\section{Histochemistry for studying structure and function of the articular disc of the human temporomandibular joint}

\author{
N. Kiga \\ Oral and Maxillofacial Surgery, Minami \\ Wakayama Medical Center, Tanabe-City, \\ Wakayama, Japan
}

\begin{abstract}
The articular disc of the temporomandibular joint (TMJ) is composed of fibrocartilage, and the extracellular matrix of this disc is composed mainly of collagen, glycosaminoglycan and proteoglycans. Research on the changes that occur in the composition of the articular disc of the TMJ is necessary for understanding the basis of the pathological process of internal derangement (ID), and a number of reports have been published in recent years on the application of refined histochemical techniques to investigate the structure and function of the TMJ. The direction of future TMJ disc studies should be towards obtaining more evidence to support previous results, and should hopefully be of practical use in terms of prevention and cure of ID.
\end{abstract}

\section{Introduction}

The articular disc of the temporomandibular joint (TMJ) is composed of fibrocartilage and the extracellular matrix of this disc is composed mainly of collagen, glycosaminoglycan and proteoglycans. ${ }^{1,2}$ Analysis of the cellular components of TMJ discs has shown the presence of fibroblast-like cells, fibrochondrocytes and chondrocyte-like cells. ${ }^{3}$ Investigation of changes in the composition of the articular TMJ disc is important for understanding the significance of the pathological process of internal derangement (ID). ${ }^{4,5}$

\section{Comments}

In the last decades of the $20^{\text {th }}$ century the presence of collagen, and the assembly and regulation of collagen fibers in the articular disc of the TMJ was investigated. ${ }^{6}$ However, few pathological changes in the articular disc of the TMJ have been investigated in recent years due to the treatment policies and ethical regulations regarding ID in individual coun- tries. A number of reports regarding the articular TMJ disc have however been published in recent years in this journal..$^{7-11}$

Although studies of the TMJ disc of adult humans have previously shown the expression of type I and III collagen in TMJ discs, $, 2,13$ studies that have analyzed the TMJ disc of human fetuses have been extremely rare. However, Moraes et al. confirmed the expression of type I and III collagen markers in the TMJ disc of human fetuses by using an immunohistochemical method. ${ }^{11}$ They further showed that type I collagen is observed in the posterior band of the articular disc and that type III collagen is observed on the inferior face of the articular disc. In conclusion, they suggested that heterotypical fibril meshes form between those two types of collagen. It is known that the TMJ disc of human fetuses grows dramatically inside the body, at which time a slight degree of stress arises inside the body due to sucking behavior. We consider that it is possible that significant changes occur in the TMJ disc of an unborn child in comparison with a post-natal child. It is therefore to be hoped that more detailed studies of the TMJ disc in human fetuses will be carried out in the future.

It is well known that hyaluronan (HA) is an important component of joints in the body and is involved in the maintenance of tissue architecture, cell proliferation, migration, differentiation, angiogenesis, wound healing and tumorigenesis. ${ }^{14} \mathrm{HA}$ is synthesized by hyaluronan synthases (HASs). Three isoforms of HAS have been shown to be responsible for the synthesis and regulation of different molecular weight HA: HAS1 and HAS2 polymerize high molecular weight $\mathrm{HA}$, whereas HAS3 generates low molecular weight HA. Matsumoto et al. have studied the relationship between HAS3 and pathological changes in TMJ discs with ID. ${ }^{9}$ Immunohistochemical analysis indicated moderate to strong staining of HAS3 in deformed TMJ discs with ID. RT-PCR experiments showed that the expression of HAS3 mRNA in these discs was significantly increased under hypoxic conditions with the addition of interleukin-1 beta (IL-1 $\beta$ ). Their study suggests that HAS3 expression is related to pathological changes in human TMJ discs affected by ID. Further examination of human TMJ disc cells would strengthen this proposition. We reported that lumican and fibromodulin, which are small, leucine-rich repeat proteoglycans, play a role in TMJ discs with ID. Lumican is associated with the assembly and regulation of collagen fibers as well as with cell migration and its expression has been reported to change in deformed TMJ discs. In deformed discs, lumican is expressed in areas of the disc that contain many fibroblast-like cells. Strong lumican expression is observed in areas that are negative for metachromasia, as
Correspondence: Prof. Norifumi Kiga, Oral and Maxillofacial Surgery, Minami Wakayama Medical Center, 27-1, Takinai-Cho, Tanabe-City, Wakayama, 646-8558, Japan.

Tel/Fax: +81.739.267050.

E-mail: nkiga@mwn.hosp.go.jp

Key words: temporomandibular joint.

Received for publication: 20 December 2011. Accepted for publication: 21 December 2011.

This work is licensed under a Creative Commons Attribution NonCommercial 3.0 License (CC BYNC 3.0).

@C Copyright N. Kiga, 2012

Licensee PAGEPress, Italy

European Journal of Histochemistry 2012; 56:e11 doi:10.4081/ejh.2012.e11

assessed by toluidine blue staining. Fibromodulin is expressed in areas with weak lumican expression and it is also expressed adjacent to the area of strong lumican expression. IL-1 $\beta$ induced a significant increase in lumican mRNA after $24 \sim 48 \mathrm{~h}$ incubation compared to the level in cells grown in the absence of IL-1 $\beta$.These findings suggest that lumican and fibromodulin are differentially regulated and that lumican may promote regeneration of the TMJ following the degeneration and deformation induced by IL-1 $\beta$.

Blood vessels are absent from normal TMJ discs but novel blood capillaries are observed to have grown and be distributed within the internal derangement of human TMJ discs. Such blood vessels may have arisen through the action of VEGF, a pro-angiogenic factor, which appears to be released by chondrocytes in hypertrophic cartilage in a paracrine way. Loreto et al. studied the expression of tumor necrosis factor-related apoptosis-inducing ligand (TRAIL), death receptor-5 (DR5) and caspase 3 in TMJ degenerated disc vessels in order to evaluate the involvement of apoptosis in angiogenesis. ${ }^{7}$ They considered that such apoptosis might form part of a self-limiting process by which the formation of new blood vessels is inhibited. High immunoexpression of TRAIL, its receptor DR5 and caspase 3 was demonstrated in the intima and media layers of newly formed vessels, in which strongly positively stained cells were observed. This research group suggested that activation of vessel apoptosis in a TMJ disc with ID could be regarded as a self-limiting process that is an attempt to lead to vessel regression. There has been no literature regarding such apoptosis in recent years. The report of Loreto et al. is therefore considered a valuable study for analysis of changes in the TMJ disc.

Human TMJ discs such as those used in the above reports are difficult to obtain for basic 
research studies, the described histochemical results are of great significance. Future studies should be performed in the attempt to obtain additional information that could be practically useful for the prevention and the cure of ID, and owing to the tight relationship between alterations of the TMJ structure and the functional impairment in ID joints, it is worth stressing that histochemistry will remain the most suitable approach for such investigations.

\section{References}

1. Rees LA. The structure and function of the mandibular joint. Br Dent J 1954;96: $125 \mathrm{e} 33$.

2. Bade H, Schenck C, Koebke J. The function of discomuscular relationships in the human temporomandibular joint. Acta Anat (Basel) 1994;151:258e67.

3. Kuroda S, Tanimoto K, Izawa T, Fujihara S, Koolstra JH, Tanaka E. Biomechanical and biochemical characteristics of the mandibular condylar cartilage. 0steoarthritis Cartilage 2009;17:1408-15.

4. Tanaka E, Detamore MS, Mercuri LG.
Degenerative disorders of the temporomandibular joint: etiology, diagnosis, and treatment. J Dent Res 2008;87:296-307.

5. Reneker J, Paz J, Petrosino C, Cook C. Diagnostic accuracy of clinical tests and signs of temporomandibular joint disorders: a systematic review of the literature. J Orthop Sports Phys Ther 2011;41:408-16.

6. Detamore MS, Athanasiou KA. Structure and function of the temporomandibular joint disc: implications for tissue engineering. J Oral Maxillofac Surg 2003;61: 494-506.

7. Loreto C, Almeida LE, Migliore MR, Caltabiano M, Leonardi R. TRAIL, DR5 and caspase 3-dependent apoptosis in vessels of diseased human temporomandibular disc. An immunohistochemical study. Eur J Histochem 2010; 54:175-179.

8. Kiga N, Tojyo I, Matsumoto T, Hiraishi Y, Shinohara Y, Fujita S. Expression of lumican related to CD34 and VEGF in the articular disc of the human temporomandibular joint. Eur J Histochem 2010;54:e34.

9. Matsumoto T, Inayama M, Tojyo I, Kiga N, Fujita S. Expression of hyaluronan synthase 3 in deformed human temporomandibular joint disc: in vivo and in vitro studies. Eur J Histochem 2010;54:e50.
10. Kiga N, Tojyo I, Matsumoto T, Hiraishi Y, Shinohara Y, Makino S, et al. Expression of lumican and fibromodulin following interleukin-1 beta stimulation of disc cells of the human temporomandibular joint. Eur J Histochem 2011;55:e11.

11. Moraes LOC, Lodi FR, Gomes TS, Marques SR, Oshima CTF, Lancellotti CLP, et al. Immunohistochemical expression of type I and III collagen antibodies in the temporomandibular joint disc of human fetuses. Eur J Histochem 2011;55:e24.

12. Salo LA, Raustia AM. Type II and type III collagen in mandibular condylar cartilage of patients with temporomandibular joint pathology. J Oral Maxillofac Surg 1995;53: $39-44$.

13. Gage JP, Virdi AS, Triffitt JT, Howlett CR, Francis MJ. Presence of type III collagen in disc attachments of human temporomandibular joints. Arch Oral Biol 1990;35: 283-8.

14. Fraser JRE, Laurent TC, Laurent UBG. Hyaluronan: its nature, distribution, functions and turnover. J Intern Med 1997;242: 27-33. 